\title{
Interaction of Melanin with Metal lons Modulates Their Cytotoxic Potential
}

\author{
Tadeusz Sarna $^{1}$ (D) $\cdot$ Harold M. Swartz ${ }^{2} \cdot$ Andrzej Zadlo $^{1}$ (D)
}

Received: 22 February 2021 / Revised: 4 June 2021 / Accepted: 3 July 2021 /

Published online: 12 July 2021

(C) The Author(s) 2021

\begin{abstract}
Melanin is one the most common biological pigments. In humans, specialized cells called melanocytes synthesize the pigment from tyrosine and 3,4-dihydroxyphenylalanine via enzyme-catalyzed reactions and spontaneous processes. The formed melanin granule consists of nanoaggregates of oligomers containing different monomers. Although the main biological function of melanin is protection against damage from solar radiation, melanin may also be involved in protection against oxidative stress. In the latter function, sequestration of redox-active metal ions and scavenging of reactive oxygen species are of importance. The paper reviews basic physicochemical properties of melanin responsible for binding of metal ions and discusses specific conditions that may induce cytotoxicity of metal ions such as iron and copper by facilitating their redox activation and release from melanin. While the value of EPR spectroscopy and other EPR-related techniques for the study of melanin is emphasized, the concomitant use of other physicochemical methods is the most efficient approach.
\end{abstract}

\section{Introduction}

Melanin is widely present in most or all complex animals. Basic building blocks of melanin are nanoaggregates of relatively small oligomers formed not only via enzymatic process but also spontaneously from the reactions of active precursors, such as dopaquinone [1-5]. So-called eumelanin is the main naturally occurring mammalian melanin pigment, responsible for brown-black coloration of pigmented tissues.

Tadeusz Sarna

tadeusz.sarna@uj.edu.pl

Harold M. Swartz

Harold.M.Swartz@dartmouth.edu

1 Department of Biophysics, Faculty of Biochemistry, Biophysics and Biotechnology, Jagiellonian University, Kraków, Poland

2 The Geisel School of Medicine at Dartmouth, Dartmouth College, Hanover, NH, USA 
Key monomer units of the eumelanin oligomers are 5,6-dihydroxyindole (DHI) and 5,6-dihydroxyindole-2-carboxylic acid (DHICA), as well as their oxidized forms [6]. Although dopachrome, one of the main intermediates of melanogenesis, spontaneously converts to DHI, the biosynthesis of eumelanin pigments involves enzymatic tautomerization of dopachrome to DHICA, which significantly increases the percentage of DHICA monomers in natural eumelanin [7]. Interestingly, the interaction of metal ions with melanin precursors could affect the result of melanogenesis. Indeed, it was shown that $\mathrm{Cu}$ (II) ions stimulated the rearrangement of dopachrome to DHICA $[8,9]$. A mechanism of dopachrome tautomerization into DHICA catalyzed by $\mathrm{Cu}(\mathrm{II})$, based on quantum chemical calculations, indicated the important role of $\mathrm{Cu}$ (II) coordination to the oxygen of quinoids of dopachrome [10]. While some of the functions of melanin, especially protection against damage from light, have evolved by natural selection, other functions seem to have arisen from the myriad physical-chemical properties intrinsic to melanin, especially interactions with metal ions and related oxidation/reduction reactions. These additional properties have been incorporated into natural defenses and also have been a potential source of pathophysiology. In this manuscript we have attempted to provide a systematic examination of these properties with a particular emphasis on how they modulate cytotoxic processes of both melanin and pathways influenced by melanin.

Melanin is very resistant to conventional chemical-physical characterization because of its insolubility in most solvents, heterogeneity of structure, and many different reactive groups $[1,2]$. These groups give rise to the redox and metal binding by melanin. Fortunately, one of the properties of these complex polymers are the presence of unpaired electrons, which can be studied non-destructively by electron paramagnetic resonance (alternatively termed electron spin resonance and usually abbreviated as EPR or ESR). In addition, further information can be obtained by the addition of paramagnetic ions to melanin using EPR to characterize their physical and chemical interactions. The senior authors of this manuscript have published more than 100 manuscripts using EPR to characterize melanin and its interactions. Therefore, we have drawn extensively but not exclusively on this body of literature to try to provide a systematic analysis of some of the relevant properties of melanin and their consequences. Although interactions of metal ions with so-called pheomelanin (a pigment derived from cysteinyldopas) are also very important, due to the fact that only limited relevant data are currently available, we decided to omit pheomelanin in this review.

\section{Key Physicochemical Properties of Eumelanin that Determine Its Interaction with Metal Ions}

Among various physicochemical properties of melanin, its ability to bind redoxactive metal ions, might be especially important biologically [11-13]. Over half a century ago it was demonstrated that melanin, interacting with metal ions, behaved as a weak acid cation exchanger [14]. Subsequent studies, focusing on determination of the molecular parameters of melanin-metal ion complexes [15-19], were to a large extent stimulated by our work that explained the unusual effect of bound to 
melanin paramagnetic metal ions on the melanin free radical signal [20]. The effect, purely physical in nature, was due to magnetic dipolar interaction between the melanin radicals and paramagnetic metal ions bound to melanin. Using selected transition metal and lanthanide ions with different spin-lattice relaxation times, and viewing melanin radicals as natural probes that enabled the detection of metal ions bound to melanin, the existence of several specific types of metal binding sites in melanin were demonstrated.

This led to the development of so-called multiprobe approach to study melanin. In this method, a suitably chosen molecular probe such as a paramagnetic metal ion (or as later shown also diamagnetic metal ions) is introduced into the melanin structure, where it is subjected to the influence of local environment and/or where it can affect redox equilibria of melanin subunits. The interaction between the probe and the local milieu of the melanin structure, detected by application of the appropriate spectroscopy, can provide unique information about the type of ligands, geometry of the complexes, and their spatial distribution.

X-band EPR spectroscopy and application of the isotope ${ }^{63} \mathrm{Cu}$ (II) to probe metalion binding sites of synthetic catechol and dopa-melanin, revealed that below pH 5.0 in both melanins only monodentate carboxyl complexes were formed, while at $\mathrm{pH}$ above 6.0 both melanins had bidentate phenolic hydroxy complexes [17]. At higher $\mathrm{pH}$, the copper ions also formed bidentate nitrogen-carboxyl complexes. While at $\mathrm{pH}$ below 5, copper complexes with natural melanin from the choroid of bovine eyes were the same as in synthetic dopa-melanin, at $\mathrm{pH}$ above 7 the corresponding EPR spectra of natural melanin suggested possible involvement of bidentate nitrogen complexes [18]. A follow-up study employing S-band EPR spectroscopy, Mossbauer spectroscopy and atomic absorption measurements, and using copper (II), iron(III) and zinc(II) as molecular probes, enabled detailed $\mathrm{pH}$-dependent determination of the binding capacity of dopa-melanin for copper and zinc ions [19]. The study also demonstrated that chemical modification of melanin, with agents blocking specific binding groups, reduced the amount of copper ions bound to melanin, with the strongest effect observed for diazomethane and dimethyl sulfate, which efficiently block phenolic hydroxy and carboxyl groups. On the other hand, acetylation and ethylation of melanin obtained by the treatment with acetic anhydride and thionyl chloride, which predominantly block the melanin amine and carboxyl groups, respectively, had much smaller effects on the copper-binding capacity of dopa-melanin. Based on Mossbauer measurements it was concluded that Fe (III) ions predominantly bound to ortho-phenolic hydroxy groups, forming high-spin complexes with distorted octahedral symmetry. A uniform distribution of metal-ion-binding sites in the melanin structure was inferred by comparing theoretical estimates with the experimentally determined quenching of the melanin radical signal by an increasing concentration of $\mathrm{Mn}(\mathrm{II})$.

On the other hand, the use of diamagnetic $\mathrm{Zn}$ (II) as a molecular probe of ionexchange properties of melanin, revealed a rapid increase in the melanin signal intensity between $\mathrm{pH} 4$ and 6, which coincided with significant uptake of zinc ions bound to melanin. The enhancement of the melanin EPR signal induced by certain multivalent diamagnetic metal ions, particularly zinc, was first reported in Ref. [21]. The molecular mechanism of this phenomenon was satisfactorily explained in Ref. 
[16]. A thorough EPR study of the effect of several dipositive alkali earth metal ions, dipositive, and tripositive transition metal and lanthanides ions on intensity, and the linewidth of the melanin radical signal revealed the formation of melanin radical-metal ion complexes. The broadening of the melanin signal depended on the nuclear spin of the metal ions indicating unresolved hyperfine splitting, consistent with a very strong interaction between melanin radicals and the metal ion. The postulated comproportionation equilibrium between melanin subunits determined the concentration of the observable free radicals. Because the interaction of metal ions with the radical is assumed to be much stronger than with either the oxidized (ortho-quinones) or reduced (ortho-hydroquinone) melanin subunit, by removing the uncomplex radical from the system, chelation of metal ions shifts the equilibrium to the right:

$$
\mathrm{o}-\mathrm{H}_{2} \mathrm{Q}+\mathrm{o}-\mathrm{Q} \leftarrow \rightarrow 2 \mathrm{H}^{+}+2 \mathrm{o}-\mathrm{SQ} ; \mathrm{o}-\mathrm{SQ}+\mathrm{M}^{\mathrm{n}+} \leftarrow \rightarrow \mathrm{M}^{\mathrm{n}+}--\mathrm{o}-\mathrm{SQ}
$$

where o- $\mathrm{H}_{2} \mathrm{Q}$ : fully reduced; o-Q: fully oxidized; o-SQ: semi-oxidized $[16,22]$

In eumelanin, the corresponding melanin subunits are 5,6-dihydroxyindole (DHI) and 5,6-dihydroxyindole-2-carboxylic acid (DHICA), their fully oxidized forms, and the corresponding free radicals.

Potentiometric titration was used in combination with EPR and absorption $\mathrm{UV}-\mathrm{V}$ is spectroscopy to quantify the acidic functionalities in a synthetic colloidal DHI-melanin [23]. The study concluded that quinone-imine functionality played a substantial role in binding of copper(II) and zinc(II) ions by DHI melanin. At neutral $\mathrm{pH}$ values, binding of metal ions was driven by quinone imine, and catechol groups yielding a mixed group of donor complexes. At slightly acidic $\mathrm{pH}$ values, lower than $6, \mathrm{Zn}$ (II) ions preferred to bind to quinone imine in 1:1 and 1:2 ratio, but $\mathrm{Cu}$ (II) retained a preference for the mixed complexation by both ligand types. At alkaline $\mathrm{pH}$ values, both metals bound to two catechol groups. Although the determined equilibrium constants for the interaction for DHI melanin- $\mathrm{Cu}$ (II) and DHI melanin-Zn(II) may be used to predict the complexation between melanin and other complexing ligands for copper and zinc ions, the study is not very representative for natural melanins, which contain significant amount of DHICA subunits $[1,2]$. Unlike natural eumelanin and synthetic melanin derived from dopa, DHI melanin has few or no carboxylic groups, which have been demonstrated to play an important role in binding of copper ions by natural eumelanin and dopa-melanin at acidic $\mathrm{pH}$ values and of alkaline earth metal ions [17-19, 24].

The role of catechol groups in binding of iron(III) by Sepia melanin was confirmed by resonance Raman spectroscopy [25]. In contrast to the natural eumelanin, $\mathrm{Fe}$ (III)-enriched samples exhibited absorption features, which were attributed to ligand-to-metal charge-transfer (LMCT). Resonance Raman spectra obtained after exciting the LMCT bands revealed bands that were assigned to Fe-OR stretching and ring deformation modes, substantiating that the Fe(III) melanin binding site is composed of catechol-like structural units. In a follow-up study, the binding capacity of Sepia melanin was compared for several alkali earth and transition metal ions [24]. The study concluded that at neutral and mildly acidic $\mathrm{pH}$ values, $\mathrm{Ca}$ (II) and $\mathrm{Mg}(\mathrm{II})$ bind to carboxyl groups of Sepia melanin with high capacity, low affinity, 
and show $\mathrm{pH}$ dependence near the $\mathrm{p} K_{\mathrm{a}}$ of the $\mathrm{COOH}$ group. In contrast, $\mathrm{Fe}(\mathrm{III})$ binds to $o$-diOH, with high capacity high affinity and no $\mathrm{pH}$ dependence over the range of $\mathrm{pH}$ examined. The data indicate that the bound metal cations are distributed throughout the melanin granules. This and the high degree of reversible metal binding may imply, according to the authors of the cited paper, the existence of ion diffusion channels within the melanin granules. The ability of iron ions to change their location within the melanin granules after initial binding, was inferred from the changes in microwave power saturation of the melanin free radical signal that gradually occurred after the binding of the iron [26].

The involvement of the catechol hydroxy, amine, and carboxylic groups in binding of monovalent and divalent alkali metal ions, divalent, and trivalent transition metal ions and lanthanide ions by natural and synthetic melanins was recently reviewed [27]. The authors emphasized that factors determining the binding sites of each metal ion are the type of eumelanin and $\mathrm{pH}$. The main features of binding of selected metal ions by melanins are compiled in Table 1. The typical effect of zinc, copper and iron ions on EPR signal of melanin radicals is shown in Fig. 1a. Although $\mathrm{Zn}(\mathrm{II})$ at high concentration is able to increase EPR signal of DOPA-melanin even 40 times, at the selected concentration $(0.09 \mathrm{mM})$, zinc increases the signal intensity by almost a factor of two. At such concentration, copper and iron ions markedly reduce the EPR signal of melanin radicals.

\section{Modulation by Melanin of the Reactivity and Availability of Metal Ions}

Iron and copper are the biologically most relevant redox-active transition metal ions. These ions, in the presence of common oxidants or reductants, can undergo reversible oxidation and reduction. As specific complexes with sulfur and nitrogen ligands, these metal ions are involved in electron transfer responsible for energy generation by the mitochondrial electron transport chain [33]. However, "free" copper and iron ions can be quite toxic due to their ability to catalyze free radical decomposition of hydrogen peroxide [34]. Of course, there are no really free metal ions in the cytoplasm for they always form complexes with available ligands such as water and low molecular weight constituents of the cell. Nevertheless, such complexes can also be involved in the so-called Fenton reaction yielding hydroxyl radicals or highly reactive metal ions in their higher oxidation state $[35,36]$. The effect of melanin on iron-associated generation of hydroxyl radicals from hydrogen peroxide was analyzed by EPR-spin trapping and absorption UV-Vis spectrophotometry [37]. The study clearly demonstrated that melanin affected the yield of hydroxyl radical in a complex way, depending on the experimental conditions. For low concentrations of $\mathrm{Fe}(\mathrm{II})$ ions, synthetic dopa-melanin dramatically reduced the yield of $\mathrm{OH}$ generation due to binding of iron to melanin. Later it was reported that even under anaerobic conditions, upon binding by melanin Fe(II) ions were oxidized to the Fe (III) state [38]. It was also shown that binding of Fe (III) ions by melanin substantially modified redox properties of the ions in that Fe (III) ions bound to melanin were much more difficult to reduce by biological reductants in comparison with "free" 


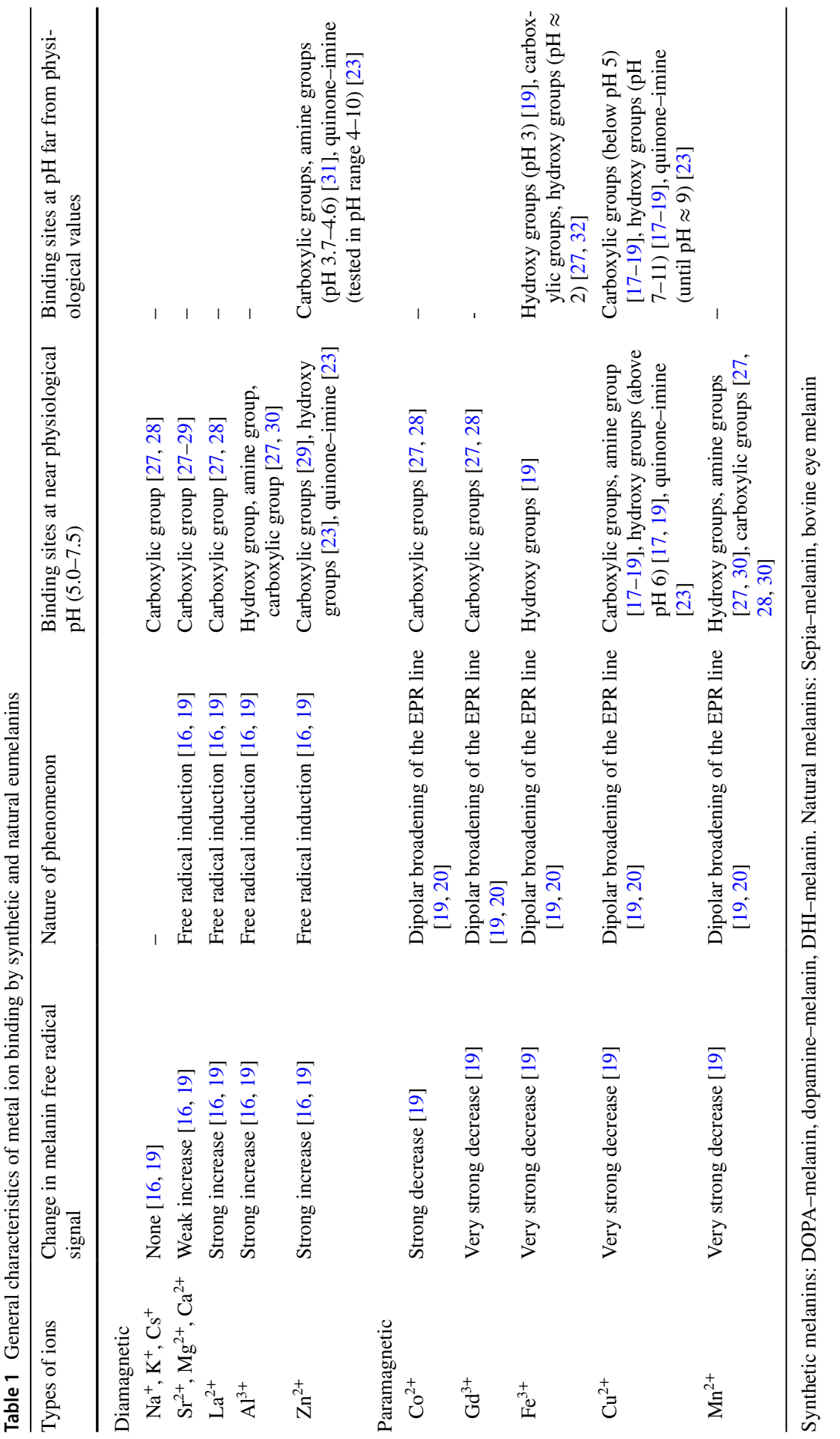



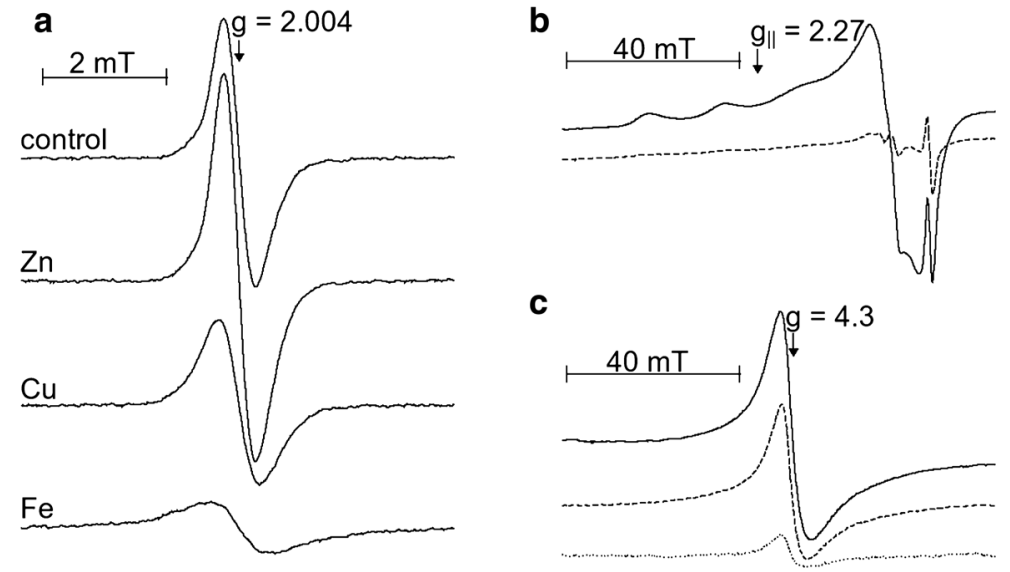

Fig. 1 EPR spectra of melanin radicals (a), copper (II) (b) and iron (III) (c) detected in samples of DOPA-melanin with and without indicated metal ions. a: melanin concentration $0.5 \mathrm{mg} / \mathrm{ml}$, metal ion concentration $0.09 \mathrm{mM}$. b and c: melanin concentration $1 \mathrm{mg} / \mathrm{ml}$, metal ion concentration $0.18 \mathrm{mM}$, rose Bengal concentration $0.5 \mathrm{mM}, \beta-\mathrm{NADH}$ concentration $2.5 \mathrm{mM}$ (except dotted line in c). Metal ion is copper (b) or iron (c). Continuous line: in dark, broken and dotted line: after 20 min. irradiation with green light $\left(516-586 \mathrm{~nm}, 50 \mathrm{~mW} / \mathrm{cm}^{2}\right)$, dotted line: $\beta-\mathrm{NADH}$ concentration $12.5 \mathrm{mM}$. All spectra recorded at $77 \mathrm{~K}$

Fe (III) ions [39]. Unlike $\mathrm{Cu}$ (II), which could be easily reduced by strong reductants, formed by a photosensitized reaction involving rose Bengal and $\beta$-NADH [40], Fe (III) bound to synthetic DOPA-melanin was much more difficult to reduce. To achieve significant reduction of $\mathrm{Fe}(\mathrm{III})$, five times greater concentration of $\beta$-NADH was needed in comparison with the concentration of $\beta-\mathrm{NADH}$ needed to reduce $\mathrm{Cu}$ (II) bound to melanin [38]. The different sensitivity of $\mathrm{Cu}$ (II) and $\mathrm{Fe}$ (III) bound to melanin to be reduced is illustrated in Fig. $1 \mathrm{~b}$ and c. On the other hand, melanin increased the rate of hydroxyl radical production if the predominant form of iron was Fe (III). The effect was due to the ability of melanin to reduce Fe (III) ions to Fe(II) ions and was particularly pronounced if Fe (III) ions were bound to strong chelators, which efficiently competed for iron with melanin or when the ions occurred in the excess in comparison to melanin [37]. The two effects, i.e., the reduction by melanin of unbound $\mathrm{Fe}$ (III) ions and oxidation of $\mathrm{Fe}$ (II) ions, accompanying their binding, could be explained by difference in the exposure of the reduced (o-quinol) and oxidized (o-quinone) melanin subunits to external reagents. The reduced subunits are expected to exhibit greater hydrophilicity, than the oxidized subunits, making them more exposed to the extragranular environment.

$\mathrm{Cu}$ (I) complexes with melanin were easily oxidized by dioxygen and hydrogen peroxide yielding hydroxyl radicals; however, almost $99 \%$ of the radicals were intercepted by the melanin [40]. This is an example of a site-specific reaction, in which reactive species formed by a system are very efficiently sequestered by the system itself. There is no doubt that site-specific formation and decay of reactive oxygen species that may occur in melanin, which by limiting the diffusion radius of the reactive species, significantly reduces their cytotoxicity. Thus, binding by melanin 
of redox-active metal ions, is a powerful protective mechanism, which reduces the availability and reactivity of the metal ions and limits the escape of formed radicals from the melanin granule.

The antioxidant action of a synthetic and natural neuromelanin was studied in a model system by monitoring the rate and extent of lipid peroxidation induced by $\mathrm{Fe}$ (II) ions and free radical initiators [41]. Although both free radical scavenging activity of melanin and its metal-ion sequestering ability were considered, it was concluded that in cells, where melanin is present as distinct pigment granules, the predominant mechanism of melanin protection against oxidative damage was sequestration of redox active metal ions, such as iron. The study provided experimental support for the postulated role of neuromelanin as a modulator of the availability and reactivity of metal ions [42, 43]. EPR spectroscopy and total reflection $\mathrm{X}$-ray fluorescence spectroscopy was used to measure paramagnetic metal ions, neuromelanin, and total metal content in nine selected areas of the human brain [44]. Clear correlation between the content of iron and its EPR spectrum was observed only for substantia nigra, which also exhibited a distinct EPR signal of neuromelanin. Binding of Fe (III) ions by neuromelanin from substantia nigra and by a synthetic neuromelanin model was studied by EPR spectroscopy in a followed up study [26]. It was demonstrated that when iron became bound to melanin this occurred preferentially by catechol hydroxy groups forming a complex with rhombic symmetry, and the iron was in the forms of high spin Fe (III) ions. Later, it was shown that intraneuronal neuromelanin buffered reactive iron and blocked iron-mediated oxidation of ascorbate and dopamine [45]. The study concluded that neuromelanin could protect against iron-mediated oxidative damage in a system modeling iron overload of brain and Parkinson's disease. The role of the dysregulation of cytosolic dopamine and reactive metal ions, most prominently iron, in pathogenesis of Parkinson's disease, was extensively discussed in a review paper [46]. The authors pointed out to the potentially protective effects of neuromelanin biosynthesis and neuromelanin itself, which when formed, could protect the pigmented neurons against irondependent oxidative stress by sequestering iron. It was emphasized that the equilibrium between iron, dopamine, and neuromelanin was crucial for cell homeostasis. Its disruption, induced by overload of neuromelanin with certain toxins and iron ions, could trigger a neurodegenerative process.

The protective effect of melanin against oxidative stress was demonstrated for the first time in cell culture by adding hydrogen peroxide to ARPE-19 cells containing porcine retinal pigment epithelium (RPE) melanosomes [47]. Following hydrogen peroxide delivery as a pulse or by continuous generation, employing glucose oxidase, greater resistance to critical concentrations of $\mathrm{H}_{2} \mathrm{O}_{2}$ was seen for cells containing melanosomes than for cells with control latex beads. A follow-up study confirmed the postulated mechanism of antioxidant action of melanin-its ability to bind metal ions [48]. Using EPR spectroscopy and the bathophenanthroline assay for determination of the iron content of melanosomes before and after loadening the cells, it was shown that the melanosomes could bind iron within living cells. The outcomes indicated retention of prebound iron and accumulation of iron by granules after iron delivery to cells via the culture medium, suggesting contributions to iron homeostasis by melanosomes. 


\section{Photodegradation of Melanin Decreases Its Metal lon Sequestering Ability and Antioxidant Capacity and Increases Prooxidant Properties of the Melanin}

Although it was demonstrated that melanin could protect pigmented cells against oxidative stress mediated by iron, an important issue that should be addressed is whether physicochemical changes of melanin that occur with aging modify antioxidant properties of the melanin and its protective ability [11]. This is particularly relevant for melanin in the retinal pigment epithelium of the human eye, where melanin is formed early during fetal development and shows little or no metabolic turnover throughout life [49, 50].

Considering the localization of RPE melanin and the high metabolic activity of the retina [51], it is apparent that this pigment can be exposed to high fluxes of intense visible light from focal irradiation, elevated oxygen concentrations, and photosensitizing molecules. These factors may lead to photochemical processes that result in chronic oxidative stress in the RPE. As a result, oxidative modification of the long-lived RPE melanin is likely to occur. Indeed, the content of melanin in human retinal pigment epithelium was shown to substantially decrease with age [52], and a more recent study demonstrated distinct chemical changes in the RPE melanin of older human donors, consistent with the observed experimental photoaging of melanin [53]. The latter study concluded that such oxidative modification of RPE melanin would reduce its metal ion binding ability.

To study in vitro age-related changes that in human RPE occur over many years, a simple model of photoaging of RPE melanin was developed, in which bovine or porcine RPE melanosomes were exposed, under controlled conditions, to high doses of visible light to induce different degree of photobleaching of the pigment granules [54-57]. Although aerobic irradiation of melanin leads to generation of superoxide anion, hydrogen peroxide [58, 59] and singlet oxygen [60-62], which might be involved in photodegradation of melanin, a recent study suggested the key role of singlet oxygen in melanin photobleaching [63].

Changes in melanin structure accompanying photobleaching of melanin modify its metal ion binding properties. It was shown that photodegradation of RPE melanosomes isolated from porcine eyes reduced their affinity to bind iron ions and their efficiency to inhibit generation of hydroxyl radicals by iron-catalyzed decomposition of hydrogen peroxide [54]. On the other hand, weak photodegradation of melanin increased the capacity of melanin to bind organic cations and significantly enhanced the effect of paramagnetic metal ions such dysprosium (III) or iron (III) bound to melanin on the spin-lattice relaxation time of melanin radicals [57, 64]. This phenomenon can be explained by morphological changes of pigment granules and structural modification of melanin nanoaggregates, enhancing the penetration of reagents into the melanin structure. Weakly degraded melanosomes exhibited a reduced ability to inhibit peroxidation of lipids induced by iron/ascorbate [55]. A similar effect was also observed in human RPE melanosomes; experimental photobleaching of the melanosomes resulted in substantial reduction of their efficiency to inhibit peroxidation of lipids induced by ADP-Fe/ 
ascorbate [65], where ADP is adenosine 5'-diphosphate. Although human RPE melanosomes obtained from older donors inhibited iron-induced peroxidation of lipids, the inhibitory effect was smaller than that in bovine RPE melanosomes, so the bovine RPE could be viewed as model of human melanosomes for the young.

It was shown that photobleached melanin, exhibiting a free radical EPR signal comparable with human RPE melanin from donors aged 80 years and older, retained some ability to bind iron. However, such iron bound to melanin could be precipitated by phosphate or replaced by zinc ions, indicating that iron binding in photobleached melanin was relatively weak and nonspecific [63, 64]. It suggests that in photobleached melanin, carboxyl groups rather than catechol hydroxy groups participate in iron binding. A change in the relative population of catechol hydroxy and carboxyl groups in photobleached melanin may result from oxidative degradation and ring opening of the melanin DHI and DHICA subunits [40]. Similar oxidative modifications of melanin may occur in human RPE due to chronic exposure of the melanin to visible light, especially its short-wavelength component, leading to significant reduction of the efficiency of antioxidant activity in melanin.

In humans neuromelanin principally occurs in the substantia nigra and locus coeruleus, and its content increases with age [66]. Although neuromelanin is not exposed to light, this polymer can be subjected to a high flux of hydrogen peroxide formed during oxidative deamination of dopamine catalyzed by monoamine oxidase [34]. In vitro studies showed that hydrogen peroxide at relatively high concentrations was able to induce oxidative degradation of melanin and that redox active metal ions such as iron and copper significantly accelerated this process [40, 45, 63]. In situ oxidative degradation of neuromelanin may be particularly important in the case of neuromelanin with accumulated high amounts of iron. The release of iron ions could occur from degradation of neuromelanin which could contribute to oxidative stress and degeneration of neurons in the substantia nigra, setting of onset of Parkinson Disease [43].

In summary, from the in vitro studies discussed, it is apparent that melanin exhibits potent antioxidant properties [37, 39, 41, 45, 54, 67]. Melanosomes isolated from human RPE and bovine RPE melanosomes protected against iron-induced, photoinduced, and photosensitized lipid peroxidation [55-57, 65]. On the other hand, melanosomes subjected to experimental photobleaching exhibited substantially reduced antioxidant and photoprotective properties [54-56, 65]. Perhaps even more remarkable was that RPE melanosomes, isolated from human and bovine eyes, became prooxidant after partial photobleaching, i.e., they accelerated iron-induced lipid peroxidation in a $\mathrm{Fe}(\mathrm{III})-\mathrm{ADP} /$ ascorbate system [55, 65]. Interestingly, photodegraded melanosomes, with a 50\% reduction of the melanin EPR signal accelerated lipid peroxidation in the presence of ascorbate even without exogenous iron. This prooxidant effect was partly reduced by desferrioxamine, indicating a role of weakly bound iron ions that had accumulated in the pigment granules in situ [55]. Similarly, photodegraded bovine RPE melanosomes were shown to accelerate light-induced peroxidation of liposomal lipids extracted from bovine retinas [56]. The extracted lipids may contain endogenous photosensitizers such as all-trans-retinal and lipid hydroperoxides that exhibit residual absorption in visible region. Photoexcitation of such lipids leads to the formation of reactive oxygen species [68] and can induce cytotoxicity 
[69]. Although it cannot be ruled out that the prooxidant action of photodegraded melanin results from its increased yield of singlet oxygen photoformation and decreased ability to quench this reactive oxygen species, the observed prooxidant action may be due to the presence of endogenous iron that becomes redox labile in photodegraded melanin, especially in the presence of hydrogen peroxide that accumulates at higher concentration during irradiation of photobleached melanosomes.

It is important to stress that RPE has metabolically active postmitotic cells, and therefore, they accumulate age-dependent changes, including the age pigment lipofuscin, a product of incomplete digestion of phagocytized photoreceptor outer segments [70, 71]. Lipofuscin exhibits substantial aerobic photoreactivity; irradiated with short wavelength visible light, it can generate singlet oxygen, superoxide anion and hydrogen peroxide [72]. These reactive oxygen species could contribute to chronic oxidative stress in the outer retina leading to tissue dysfunction over time that predisposes to the development of degenerative processes, such as age-related macular degeneration [73, 74]. One of the key roles of RPE cells is phagocytosis of photoreceptor outer segments membranes, which are rich in polyunsaturated fatty acids [70]. Thus, RPE lipofuscin granules also contain high amounts of polyunsaturated fatty acids. RPE melanosomes, by fusion with lipofuscin, form complex granules called melanolipofuscin [75]. It can be postulated that in such a system lipid peroxides can oxidatively modify melanin and that this process can be accelerated by redox active transition metal ions bound to the melanin. To study such interactions, a simplified model system was used, in which tert-butyl hydroperoxide (TBH) was used as a model organic peroxide resembling lipid hydroperoxide [76]. TBH was shown to induce chemical degradation of both synthetic eumelanin and melanosomes isolated from bovine RPE. Although zinc bound to synthetic melanin had no significant effect on the kinetics of such degradation, copper and iron accelerated this process and iron was more effective than copper. The higher efficiency of iron to mediate the degradation of melanin could be due to a more efficient oxidation of melanin induced by iron. These transition metal ions had negligible effect on antioxidant properties of examined nondegraded melanins. However, copper-enriched and iron-enriched melanins degraded by $\mathrm{TBH}$ accelerated light-induced peroxidation of liposomal lipids extracted from bovine retinas. Such pro-oxidant effects increased with the degree of melanin degradation. The data suggest that iron, weakly bound to carboxyl groups originating from destruction of DHI and DHICA subunits, is able to induce a Fenton type reaction. In the absence of melanin or in the presence of degraded melanin without metal ions, lipid peroxidation decreased to zero after cessation of irradiation. On the other hand, in the sample with lipids and degraded melanin with iron, lipid peroxidation continued even after the termination of irradiation, which indicates that such degraded melanin exhibits decreased ability to scavenge free radicals. It can be assumed that melanolipofuscin formed in the aging RPE provides conditions that facilitate oxidative modification of the melanin component, which may increase the risk of oxidative stress in the outer retina.

Binding of metal ions to melanin can affect aerobic reactivity of the melanin, as shown by examining the ability of Sepia melanin with several different metal ions to cause strand breaks in supercoiled pUC18 DNA [77]. The study reported that $\mathrm{Cu}(\mathrm{II})$ and $\mathrm{Fe}(\mathrm{III})$ enriched melanins induced the greatest amount of DNA damage. 
Interestingly, while EDTA reduced the amount of DNA damage induced by $\mathrm{Cu}$ (II)enriched melanin, the damaging effect of Fe(III)-enriched melanin was actually higher in the presence of this chelator. Although the exact mechanism of this phenomenon remains unknown, an independent study suggested that binding of divalent copper and zinc ions to melanin, could induce a pro-oxidant response under oxygen, generating superoxide anion and hydroxyl radicals, as shown by EPR-spin trapping [78]. In the same work, the authors observed similar pro-oxidant behavior in melanoma cell lines under external peroxide stress.

Copper ions complexed with synthetic dopa-melanin accelerated degradation of the melanin induced by a high concentration of hydrogen peroxide both in the dark and upon irradiation with UVA-Vis light [40]. The possible involvement of hydroxyl radicals, generated by free radical decomposition of hydrogen peroxide catalyzed by copper-melanin complexes, was inferred from the detection of salicylate hydroxylation products, using salicylate as a molecular probe. A similar conclusion about the catalytic role of iron bound to neuromelanin in its degradation, induced by hydrogen peroxide, was reached based on a study, in which neuromelanin isolated from human substantia nigra before and after treatment with deferoxamine was exposed to high concentration of $\mathrm{H}_{2} \mathrm{O}_{2}$ [45]. It was concluded that neuromelanin containing higher amount of iron was degraded by hydrogen peroxide with higher rate. Using EPR spectroscopy, EPR-oximetry, UV-Vis absorption spectroscopy, atomic force microscopy and dynamic light scattering, oxidative changes in synthetic dopamelanin and bovine RPE melanosomes induced by tertbutyl hydroperoxide, were analyzed [76]. The study revealed that copper and iron ions bound to melanin accelerated the degradation of the melanin, confirming the metal ion pro-oxidant action.

\section{Conclusions}

In addition to the information summarized in the various sections above, it is also possible to draw some over-arching conclusions for this review.

1. This review makes clear the value of EPR for the study of melanin. The use of EPR has contributed to an understanding of the biological properties in several important and sometimes unique ways. The value stems in part from the versatility of EPR for this application, because it can follow both the free radical of melanin and the magnetic state of metal ions and in addition their interactions. No other technique could make these measurements. There is further added value because of the resistance of melanin to characterization by many of the usual physical-chemical approaches.

2. While the EPR studies were quite valuable in themselves, the significance of many of the results was significantly advanced by the concomitant use of other techniques, such as electrochemical detection of salicylate hydroxylation products and lipid hydroperoxides, UV-Vis, absorption and emission spectroscopy, IR absorption and resonance Raman spectroscopy, potentiometric titration, atomic force microscopy and dynamic light scattering, energy dispersive X-ray analysis, total reflection X-ray spectroscopy, and various cellular and molecular biology 
techniques. In general, this is an important way to most effectively use EPR, building on it strengths while recognizing the limitations of the information that is obtained.

3. Some of the key interactions of melanin with metal ions e.g., the types of binding that occurred, were able to be resolved using model melanins with a known set of reactive groups. In general, the use of model systems can be very valuable for understanding EPR studies. But also, as is the case with melanin interactions with metals, the use of only models may not provide unambiguous information for the more complex naturally occurring biological structures.

4. This review has shown how melanins can impact the availability and reactivity of metal ions, in ways that impact biological effects. In particular are the studies on the RPE, where it has been shown that the interaction of melanin and metal ions appears to explain some of the known pathophysiology of aging eyes.

5. While the pathways from changes in the redox properties of melanin are not as well documented as the interactions with metals, the bulk of the evidence appears to show a relationship between alterations in the redox properties of melanin and possible pathophysiology. This is particularly apparent in case of melanin photoaging that changes redox equilibrium of the melanin subunits by reversible and nonreversible oxidation [53, 79-82].

6. While much has already been learned, there remain many areas of importance that need further investigation. One of them is the elucidation of the exact role of photoreactivity of the skin melanin in adverse reactions that could contribute to melanoma pathogenesis. This is an important issue for it has been shown that in experimental animals melanoma induction by ultraviolet A $(320-400 \mathrm{~nm})$ requires the presence of melanin pigment and is associated with oxidative DNA damage within melanocytes [83]. Another intriguing issue that needs to be resolved is the mechanism of energy transfer from melanin nanoaggregates (excited with UVA) to DNA nucleotides, postulated to be responsible for the dark formation of cyclobutane pyrimidine dimers, which are typically formed upon excitation of the DNA nucleotides with short wavelength UVB (290-320 nm) [84].

Funding TS and AZ support: Poland National Science Centre (Grant OPUS 16 2017/27/B/ST5/02631).

Availability of data and materials Not Applicable.

Code availability Not Applicable.

Declarations

Conflict of interest No conflict of interest.

Ethical approval Not Applicable.

Consent to participate All co-authors give consent to participate in this study.

Consent for publication All co-authors express their consent for publication. 
Open Access This article is licensed under a Creative Commons Attribution 4.0 International License, which permits use, sharing, adaptation, distribution and reproduction in any medium or format, as long as you give appropriate credit to the original author(s) and the source, provide a link to the Creative Commons licence, and indicate if changes were made. The images or other third party material in this article are included in the article's Creative Commons licence, unless indicated otherwise in a credit line to the material. If material is not included in the article's Creative Commons licence and your intended use is not permitted by statutory regulation or exceeds the permitted use, you will need to obtain permission directly from the copyright holder. To view a copy of this licence, visit http://creativecommons.org/licen ses/by/4.0/.

\section{References}

1. M. d'Ischia, K. Wakamatsu, A. Napolitano, S. Briganti, J.-C. Garcia-Borron, D. Kovacs, P. Meredith, A. Pezzella, M. Picardo, T. Sarna, J.D. Simon, S. Ito, Pigment Cell Melanoma Res. 26, 616 (2013). https://doi.org/10.1111/pcmr.12121

2. M. d'Ischia, K. Wakamatsu, F. Cicoira, E. Di Mauro, J.C. Garcia-Borron, S. Commo, I. Galván, G. Ghanem, K. Kenzo, P. Meredith, A. Pezzella, C. Santato, T. Sarna, J.D. Simon, L. Zecca, F.A. Zucca, A. Napolitano, S. Ito, Pigment Cell Melanoma Res. 28, 520 (2015). https://doi.org/10. $1111 /$ pcmr. 12393

3. S. Ito, K. Wakamatsu, Photochem. Photobiol. 84, 582 (2008). https://doi.org/10.1111/j.17511097.2007.00238.x

4. S. Ito, M. Sugumaran, K. Wakamatsu, Int. J. Mol. Sci. (2020). https://doi.org/10.3390/ijms2 1176080

5. K. Wakamatsu, J.H. Zippin, S. Ito, Pigment Cell Melanoma Res. (2021). https://doi.org/10.1111/ pcmr. 12970

6. S. Ito, K. Wakamatsu, Pigment Cell Res. 16, 523 (2003). https://doi.org/10.1034/j.1600-0749. 2003.00072.X

7. V.J. Hearing, in Pigment. Syst. Physiol. Pathophysiol., ed. by J.J. Nordlund, R.E. Boissy, V.J. Hearing, R.A. King, W.S. Oetting, J.-P. Ortonne (Blackwell Publishing Ltd, Malden, 2006), pp. 191-212

8. A. Palumbo, F. Solano, G. Misuraca, P. Aroca, J.C. Garcia Borron, J.A. Lozano, G. Prota, Biochim. Biophys. Acta 1115, 1 (1991). https://doi.org/10.1016/0304-4165(91)90003-Y

9. S. Ito, N. Suzuki, S. Takebayashi, S. Commo, K. Wakamatsu, Pigment Cell Melanoma Res. 26, 817 (2013). https://doi.org/10.1111/pcmr.12137

10. R. Kishida, A.G. Saputro, H. Kasai, Biochim. Biophys. Acta 1850, 281 (2015). https://doi.org/10. 1016/j.bbagen.2014.10.024

11. T. Sarna, J. Photochem. Photobiol. B 12, 215 (1992)

12. T.J. Sarna, H.A. Swartz, in Pigment. Syst. Physiol. Pathophysiol., ed. by J.J. Nordlund, E. Boissy Raymond, J. Hearing Vincent, A. King Richard, S. Oetting William, O. Jean-Paul (Blackwell Publishing Ltd, Malden, 2006), pp. 311-341

13. P. Meredith, T. Sarna, Pigment Cell Res. 19, 572 (2006). https://doi.org/10.1111/j.1600-0749.2006. 00345.x

14. F.W. Bruenger, B.J. Stover, D.R. Atherton, Radiat. Res. 32, 1 (1967). https://doi.org/10.2307/35723 00

15. B. Larsson, H. Tjälve, Biochem. Pharmacol. 28, 1181 (1979). https://doi.org/10.1016/00062952(79)90326-5

16. C.C. Felix, J.S. Hyde, T. Sarna, R.C. Sealy, J. Am. Chem. Soc. 100, 3922 (1978). https://doi.org/10. 1021/ja00480a044

17. W. Froncisz, T. Sarna, J.S. Hyde, Arch. Biochem. Biophys. 202, 289 (1980). https://doi.org/10. 1016/0003-9861(80)90430-0

18. T. Sarna, W. Froncisz, J.S. Hyde, Arch. Biochem. Biophys. 202, 304 (1980). https://doi.org/10. 1016/0003-9861(80)90431-2

19. T. Sarna, W. Korytowski, M. Pasenkiewicz-Gierula, E. Gudowska, in Proc. 11th Int. Pigment Cell Conf. Sendai. ed. by M. Seiji (University of Tokyo Press, Tokyo, 1981), pp. 23-29 
20. T. Sarna, J.S. Hyde, H.M. Swartz, Science 192, 1132 (1976). https://doi.org/10.1126/science. 179142

21. T. Sarna, S. Lukiewicz, Folia Histochem. Cytochem. (Krakow) 10, 265 (1972)

22. T. Sarna, P.M. Plonka, in Biomed. EPR Part Free Radic. Met. Med. Physiol., ed. by S.R. Eaton, G.R. Eaton, L.J. Berliner (Springer US, Boston, 2005), pp. 125-146

23. B. Szpoganicz, S. Gidanian, P. Kong, P. Farmer, J. Inorg. Biochem. 89, 45 (2002). https://doi.org/10. 1016/s0162-0134(01)00406-8

24. Y. Liu, L. Hong, V.R. Kempf, K. Wakamatsu, S. Ito, J.D. Simon, Pigment Cell Res. 17, 262 (2004). https://doi.org/10.1111/j.1600-0749.2004.00140.x

25. A. Samokhvalov, Y. Liu, J.D. Simon, Photochem. Photobiol. 80, 84 (2004). https://doi.org/10.1562/ 2004-01-18-RA-047.1

26. T. Shima, T. Sarna, H.M. Swartz, A. Stroppolo, R. Gerbasi, L. Zecca, Free Radic. Biol. Med. 23, 110 (1997). https://doi.org/10.1016/s0891-5849(96)00623-5

27. E.D. Mauro, R. Xu, G. Soliveri, C. Santato, MRS Commun. 7, 141 (2017). https://doi.org/10.1557/mrc. 2017.33

28. B. Larsson, H. Tjälve, Acta Physiol. Scand. 104, 479 (1978). https://doi.org/10.1111/j.1748-1716.1978. tb06303.x

29. L. Hong, J.D. Simon, Photochem. Photobiol. 81, 517 (2005). https://doi.org/10.1562/ 2005-03-02-RA-453

30. S. Aime, M. Botta, I. Camurati, J. Inorg. Biochem. 36, 1 (1989). https://doi.org/10.1016/0162-0134(89) 80008-x

31. L. Hong, J.D. Simon, Photochem. Photobiol. 82, 1265 (2006). https://doi.org/10.1562/ 2006-02-23-RA-809

32. L. Bardani, M.G. Bridelli, M. Carbucicchio, P.R. Crippa, Biochim. Biophys. Acta BBA Gen. Subj. 716, 8 (1982). https://doi.org/10.1016/0304-4165(82)90196-9

33. L.A. Sazanov, Nat. Rev. Mol. Cell Biol. 16, 375 (2015). https://doi.org/10.1038/nrm3997

34. B. Halliwell, J.M.C. Gutteridge, Free Radicals in Biology and Medicine (Oxford University Press, Oxford, 2015)

35. M. Pettine, F.J. Millero, Limnol. Oceanogr. 35, 730 (1990). https://doi.org/10.4319/lo.1990.35.3.0730

36. R.R. Crichton, S. Wilmet, R. Legssyer, R.J. Ward, J. Inorg. Biochem. 91, 9 (2002). https://doi.org/10. 1016/s0162-0134(02)00461-0

37. B. Pilas, T. Sarna, B. Kalyanaraman, H.M. Swartz, Free Radic. Biol. Med. 4, 285 (1988). https://doi. org/10.1016/0891-5849(88)90049-4

38. A.C. Żądło, T. Sarna, Acta Biochim. Pol. 66, 459 (2019). https://doi.org/10.18388/abp.2019_2889

39. M. Zareba, A. Bober, W. Korytowski, L. Zecca, T. Sarna, Biochim. Biophys. Acta 1271, 343 (1995). https://doi.org/10.1016/0925-4439(95)00058-C

40. W. Korytowski, T. Sarna, J. Biol. Chem. 265, 12410 (1990). https://doi.org/10.1016/S0021-9258(19) $38362-0$

41. W. Korytowski, T. Sarna, M. Zareba, Arch. Biochem. Biophys. 319, 142 (1995). https://doi.org/10. 1006/abbi.1995.1276

42. H.M. Swartz, T. Sarna, L. Zecca, Ann. Neurol. 32(Suppl), S69 (1992). https://doi.org/10.1002/ana. 410320712

43. W.S. Enochs, T. Sarna, L. Zecca, P.A. Riley, H.M. Swartz, J. Neural Transm. Park. Dis. Dement. Sect. 7, 83 (1994). https://doi.org/10.1007/BF02260963

44. L. Zecca, T. Shima, A. Stroppolo, C. Goj, G.A. Battiston, R. Gerbasi, T. Sarna, H.M. Swartz, Neuroscience 73, 407 (1996). https://doi.org/10.1016/0306-4522(96)00047-4

45. L. Zecca, L. Casella, A. Albertini, C. Bellei, F.A. Zucca, M. Engelen, A. Zadlo, G. Szewczyk, M. Zareba, T. Sarna, J. Neurochem. 106, 1866 (2008). https://doi.org/10.1111/j.1471-4159.2008.05541.x

46. F.A. Zucca, J. Segura-Aguilar, E. Ferrari, P. Muñoz, I. Paris, D. Sulzer, T. Sarna, L. Casella, L. Zecca, Prog. Neurobiol. 155, 96 (2017). https://doi.org/10.1016/j.pneurobio.2015.09.012

47. J.M. Burke, P. Kaczara, C.M.B. Skumatz, M. Zareba, M.W. Raciti, T. Sarna, Mol. Vis. 17, 2864 (2011)

48. P. Kaczara, M. Zaręba, A. Herrnreiter, C.M.B. Skumatz, A. Ządło, T. Sarna, J.M. Burke, Pigment Cell Melanoma Res. 25, 804 (2012). https://doi.org/10.1111/pcmr.12008

49. M. Boulton, in Retin. Pigment Epithelium Funct. Dis., ed. by F. Marmor, T.J. Wolfensberger (Oxford University Press, New York, 1998), pp. 68-85

50. U. Schraermeyer, K. Heimann, Pigment Cell Res. 12, 219 (1999). https://doi.org/10.1111/j.1600-0749. 1999.tb00755.x

51. E. R. Berman, Biochemistry of the Eye (Springer Science \& Business Media, 1991) 
52. T. Sarna, J.M. Burke, W. Korytowski, M. Rózanowska, C.M.B. Skumatz, A. Zareba, M. Zareba, Exp. Eye Res. 76, 89 (2003). https://doi.org/10.1016/S0014-4835(02)00247-6

53. S. Ito, A. Pilat, W. Gerwat, C.M.B. Skumatz, M. Ito, A. Kiyono, A. Zadlo, Y. Nakanishi, L. Kolbe, J.M. Burke, T. Sarna, K. Wakamatsu, Pigment Cell Melanoma Res. 26, 357 (2013). https://doi.org/10.1111/ pcmr. 12078

54. M. Zareba, G. Szewczyk, T. Sarna, L. Hong, J.D. Simon, M.M. Henry, J.M. Burke, Photochem. Photobiol. 82, 1024 (2006). https://doi.org/10.1562/2006-03-08-RA-836

55. A. Zadlo, M.B. Rozanowska, J.M. Burke, T.J. Sarna, Pigment Cell Res. 20, 52 (2007). https://doi.org/ 10.1111/j.1600-0749.2006.00350.x

56. A. Zadlo, J.M. Burke, T. Sarna, Photochem. Photobiol. Sci. Off. J. Eur. Photochem. Assoc. Eur. Soc. Photobiol. 8, 830 (2009). https://doi.org/10.1039/b901820d

57. A. Zadlo, G. Szewczyk, M. Sarna, A. Kozinska, A. Pilat, P. Kaczara, T. Sarna, Free Radic. Biol. Med. 97, 320 (2016). https://doi.org/10.1016/j.freeradbiomed.2016.06.012

58. C.C. Felix, J.S. Hyde, T. Sarna, R.C. Sealy, Biochem. Biophys. Res. Commun. 84, 335 (1978). https:// doi.org/10.1016/0006-291x(78)90175-4

59. W. Korytowski, B. Pilas, T. Sarna, B. Kalyanaraman, Photochem. Photobiol. 45, 185 (1987). https://doi. org/10.1111/j.1751-1097.1987.tb05362.x

60. O. Chiarelli-Neto, C. Pavani, A.S. Ferreira, A.F. Uchoa, D. Severino, M.S. Baptista, Free Radic. Biol. Med. 51, 1195 (2011). https://doi.org/10.1016/j.freeradbiomed.2011.06.013

61. S. Ito, M. Kikuta, S. Koike, G. Szewczyk, M. Sarna, A. Zadlo, T. Sarna, K. Wakamatsu, Pigment Cell Melanoma Res. 29, 340 (2016). https://doi.org/10.1111/pcmr.12469

62. G. Szewczyk, A. Zadlo, M. Sarna, S. Ito, K. Wakamatsu, T. Sarna, Pigment Cell Melanoma Res. 29, 669 (2016). https://doi.org/10.1111/pcmr.12514

63. A. Żądło, S. Ito, M. Sarna, K. Wakamatsu, K. Mokrzyński, T. Sarna, Photochem. Photobiol. Sci. (2020). https://doi.org/10.1039/C9PP00481E

64. A.C. Żąłło, Acta Biochim. Pol. 66, 237 (2019). https://doi.org/10.18388/abp.2018_2802

65. B. Rózanowski, J.M. Burke, M.E. Boulton, T. Sarna, M. Rózanowska, Invest. Ophthalmol. Vis. Sci. 49, 2838 (2008). https://doi.org/10.1167/iovs.08-1700

66. L. Zecca, F.A. Zucca, H. Wilms, D. Sulzer, Trends Neurosci. 26, 578 (2003). https://doi.org/10.1016/j. tins.2003.08.009

67. E.S. Krol, D.C. Liebler, Chem. Res. Toxicol. 11, 1434 (1998). https://doi.org/10.1021/tx980114c

68. A. Koscielniak, M. Serafin, M. Duda, T. Oles, A. Zadlo, A. Broniec, O. Berdeaux, S. Gregoire, L. Bretillon, T. Sarna, A. Pawlak, Cell Biochem. Biophys. 75, 443 (2017). https://doi.org/10.1007/ s12013-017-0832-3

69. A.M. Pawlak, M. Olchawa, A. Koscielniak, A. Zadlo, A. Broniec, T. Oles, T.J. Sarna, Eur. J. Lipid Sci. Technol. 121, 1800476 (2019). https://doi.org/10.1002/ejlt.201800476

70. O. Strauss, Physiol. Rev. 85, 845 (2005). https://doi.org/10.1152/physrev.00021.2004

71. A. Höhn, T. Grune, Redox Biol. 1, 140 (2013). https://doi.org/10.1016/j.redox.2013.01.006

72. M. Rózanowska, J. Jarvis-Evans, W. Korytowski, M.E. Boulton, J.M. Burke, T. Sarna, J. Biol. Chem. 270, 18825 (1995). https://doi.org/10.1074/jbc.270.32.18825

73. S. Beatty, H. Koh, M. Phil, D. Henson, M. Boulton, Surv. Ophthalmol. 45, 115 (2000). https://doi.org/ 10.1016/s0039-6257(00)00140-5

74. J. Cai, K.C. Nelson, M. Wu, P. Sternberg, D.P. Jones, Prog. Retin. Eye Res. 19, 205 (2000). https://doi. org/10.1016/s1350-9462(99)00009-9

75. L. Feeney-Burns, Curr. Top. Eye Res. 2, 119 (1980)

76. A. Zadlo, A. Pilat, M. Sarna, A. Pawlak, T. Sarna, Cell Biochem. Biophys. 75, 319 (2017). https://doi. org/10.1007/s12013-017-0793-6

77. L. Hong, Y. Liu, J.D. Simon, Photochem. Photobiol. 80, 477 (2004). https://doi.org/10.1562/00318655(2004)080\%3c0477:BOMITM\%3e2.0.CO;2

78. P.J. Farmer, S. Gidanian, B. Shahandeh, A.J. Di Bilio, N. Tohidian, F.L. Meyskens, Pigment Cell Res. 16, 273 (2003). https://doi.org/10.1034/j.1600-0749.2003.00046.x

79. M. Zareba, T. Sarna, G. Szewczyk, J.M. Burke, Photochem. Photobiol. 83, 925 (2007). https://doi.org/ 10.1111/j.1751-1097.2007.00080.x

80. B. Rózanowski, J. Cuenco, S. Davies, F.A. Shamsi, A. Zadło, P. Dayhaw-Barker, M. Rózanowska, T. Sarna, M.E. Boulton, Photochem. Photobiol. 84, 650 (2008). https://doi.org/10.1111/j.1751-1097.2007. 00259.x

81. A. Zadlo, G. Szewczyk, M. Sarna, T.G. Camenisch, J.W. Sidabras, S. Ito, K. Wakamatsu, F. Sagan, M. Mitoraj, T. Sarna, Pigment Cell Melanoma Res. 32, 359 (2019). https://doi.org/10.1111/pcmr.12752 
82. M.M. Olchawa, G.M. Szewczyk, A.C. Zadlo, O.I. Krzysztynska-Kuleta, T.J. Sarna, Pigment Cell Melanoma Res. (2020). https://doi.org/10.1111/pcmr.12914

83. F.P. Noonan, M.R. Zaidi, A. Wolnicka-Glubisz, M.R. Anver, J. Bahn, A. Wielgus, J. Cadet, T. Douki, S. Mouret, M.A. Tucker, A. Popratiloff, G. Merlino, E.C. De Fabo, Nat. Commun. 3, 884 (2012). https:// doi.org/10.1038/ncomms1893

84. S. Premi, S. Wallisch, C.M. Mano, A.B. Weiner, A. Bacchiocchi, K. Wakamatsu, E.J.H. Bechara, R. Halaban, T. Douki, D.E. Brash, Science 347, 842 (2015). https://doi.org/10.1126/science.1256022

Publisher's Note Springer Nature remains neutral with regard to jurisdictional claims in published maps and institutional affiliations. 